\title{
La confrérie de la Sainte-Famille à Québec sous le régime français, 1663-1760
}

\section{Marie-Aimée Cliche}

Volume 43, 1976

URI : https://id.erudit.org/iderudit/1007231ar

DOI : https://doi.org/10.7202/1007231ar

Aller au sommaire du numéro

Éditeur(s)

Les Éditions Historia Ecclesiæ Catholicæ Canadensis Inc.

ISSN

0318-6172 (imprimé)

1927-7067 (numérique)

Découvrir la revue

Citer cet article

Cliche, M.-A. (1976). La confrérie de la Sainte-Famille à Québec sous le régime français, 1663-1760. Sessions d'étude - Société canadienne d'histoire de l'Église catholique, 43, 79-93. https://doi.org/10.7202/1007231ar

Tous droits réservés ( Les Éditions Historia Ecclesiæ Catholicæ Canadensis Inc., 1977
Ce document est protégé par la loi sur le droit d'auteur. L’utilisation des services d'Érudit (y compris la reproduction) est assujettie à sa politique d'utilisation que vous pouvez consulter en ligne.

https://apropos.erudit.org/fr/usagers/politique-dutilisation/ 


\section{La confrérie de la Sainte-Famille à Québec} sous le régime français, $1663-1760$

La dévotion à la sainte Famille trouve son origine dans l'École de spiritualité française du XVIIe siècle. Les grands mystiques comme Bérulle, Bourgoing et Olier donnèrent à cette dévotion un caractère essentiellement théocentrique, les personnes de Marie et Joseph n'ayant de l'importance que rattachées au Verbe Incarné. Les jésuites joignirent à la sainte Famille le culte des saints Anges qu'ils répandirent largement dans leurs congrégations de la Vierge.

Cette dévotion atteignit la Nouvelle-France par l'intermédiaire de la Société Notre-Dame de Montréal. Les membres de cette société avaient dédié la ville de Montréal à la sainte Famille en 1642 et Jérôme Le Royer de La Dauversière avait fondé une confrérie de la Sainte-Famille à Laflèche en 1632.

Lors de la dissolution de la Société en 1663, l'un des membres, madame Barbe de Boullogne, femme de Louis d'Ailleboust, conçut le projet de fonder une confrérie dédiée à la sainte Famille du Verbe Incarné, destinée à promouvoir la sanctification des familles. L'esprit de cette confrérie serait d'amener les participants à " faire partie de la sainte Famille » en imitant "la personne que chacun représente ${ }^{1}$ ", les pères de famille devant acquérir les vertus de saint Joseph, les mères, celles de Marie, les enfants, la perfection de l'enfant Jésus, les serviteurs, le dévouement des saints Anges.

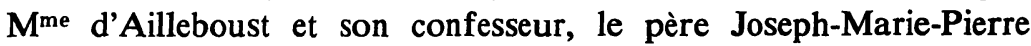
Chaumonot (lui-même un grand dévot de la sainte Famille, comme tous les missionnaires jésuites de la Nouvelle-France) s'unirent au sulpicien Gabriel Souart, à Marguerite Bourgeois, Jeanne Mance et Judith Moreau de Brésoles pour fonder leur confrérie.

Mis au courant de ce projet, $\mathbf{M}^{\mathrm{gr}}$ François de Laval invita les deux innovateurs à venir organiser la même association à Québec. Ancien disciple des jésuites et membre de la Congrégation de la

1 Pierre Chaumonot, Autobiographie, Paris, Oudin, 1885, pp. 168-169. 
Vierge, $\mathbf{M}^{\mathrm{gr}}$ de Laval participait à cette dévotion à la sainte Famille à qui il avait dédié son Séminaire.

Grâce à une collaboration étroite entre les dévots de Montréal, un jésuite et le premier évêque du Canada, la confrérie de la Sainte-Famille s'élabora sous sa forme définitive et fut érigée solennellement par un mandement le 14 mars $1664^{2}$.

\section{I - ESPRIT DE LA CONFRÉRIE : CONSTITUER UN CORPS D'ÉLITE}

$M^{\text {gr }}$ de Laval rêvait de voir fleurir au Canada les vertus de la primitive Église. Pour assurer la sanctification de ses ouailles, il voulut placer sous leurs yeux des exemples d'une vie chrétienne parfaite, puisés chez des gens non consacrés à Dieu par vocation. Ces corps d'élite, comme la Congrégation de la Vierge et la confrérie de la Sainte-Famille, seraient caractérisés par la qualité morale de leurs membres, la sévérité des règlements et des conditions d'admission. Ces associations contribueraient à fournir l'exemple vivant que l'Église voulait projeter de l'idéal à imiter.

\section{A - Élite Morale}

\section{Qualité des membres}

Les membres fondateurs de la confrérie de la Sainte-Famille possédaient à peu près toutes les qualités requises pour constituer des exemples de vie chrétienne parfaite. $\mathbf{M}^{\mathrm{gr}}$ de Laval, $\mathbf{M}^{\mathrm{me}} \mathrm{d}^{\text {'Aille- }}$ boust, $\mathbf{M}^{\mathrm{me}}$ Bourdon jouissaient tous trois d'une enviable réputation de sainteté de leur vivant même. Sous leur impulsion, la confrérie de la Sainte-Famille devint une école de ferveur et parfois même de sainteté pour quelques-unes des personnes affiliées.

Par exemple, Marie Favery, la fondatrice de la confrérie du Rosaire; Jeanne Le Ber, la célèbre recluse de Montréal; $M^{\text {me }}$ d'Youville, la fondatrice des Sœurs Grises; $\mathbf{M}^{\mathrm{me}}$ de Vaudreuil, la femme du dernier gouverneur du Canada; $M^{\text {me }}$ de Champigny, l'épouse de l'intendant, qui édifiait tout le monde par sa charité et sa piété; Catherine Tekakouitha, Iroquoise de la mission SaintFrançois-Xavier.

2 Mandements des évêques de Québec, Vol. I, Québec, 1887, pp. 5166. 


\section{Sévérité des règlements}

En plus de suivre l'exemple édifiant des fondateurs, les membres de la confrérie étaient maintenus dans la voie de la perfection par la sévérité des règlements, la haute spiritualité de l'association.

Le but de la confrérie consistait à amener les membres à faire «une profession plus particulière des vertus chrétiennes que les autres personnes du monde ${ }^{3}$ ». Les règlements exigeaient donc un plus grand nombre d'actes de piété extérieurs et une observance scrupuleuse des volontés de l'évêque.

a) Exercices de piété calqués sur la congrégation

Les exercices de piété prescrits aux membres de la SainteFamille étaient calqués sur ceux de la congrégation mariale dirigée par les jésuites. L'idée venait de $\mathbf{M}^{\mathrm{gr}}$ de Laval. Pour éviter que le recrutement d'une association nuise à l'autre, il avait décrété que la Congrégation serait réservée aux hommes tandis que la Sainte-Famille n'accueillerait que des femmes. La spiritualité des deux associations serait la même, les exercices de piété identiques, plus quelques pratiques destinées à mettre en évidence la dévotion à la sainte Famille, comme d'en afficher une image dans les maisons, réciter le chapelet de la Sainte-Famille, lire un chapitre de son manuel.

\section{b) Morale austère}

Les règles de morale imposées aux dames de la Sainte-Famille différaient un peu de celles de la Congrégation parce qu'adaptées à une clientèle féminine.

Le Manuel de la Solide Dévotion à la sainte Famille prône un type de dévotion austère bien caractéristique du XVII ${ }^{e}$ siècle français .Toutes les femmes qui le désirent sont cependant admissibles dans la confrérie, à condition de manifester une dévotion particulière à la sainte Famille, de faire preuve de bonne volonté à se corriger de leurs défauts et de n'être pas scandaleuses. Comme les dames de cette confrérie doivent servir d'exemple au reste de la population, on conçoit aisément que le scandale soit le péché le plus opposé à l'esprit de la Sainte-Famille.

3 Ango Des Mazerets, La solide dévotion à la très sainte Famille de Jésus, Marie et Joseph avec un catéchisme qui enseigne à pratiquer leurs vertus, Paris, Florentin Lambert, 1675, p. 87. 
La principale forme de scandale à éviter consistait dans tout défaut un peu notable contre la chasteté. Pour pratiquer cette vertu, les dames de la Sainte-Famille devaient garder la plus grande modestie dans leurs vêtements, s'abstenir complètement d'aller au bal ou à des réunions mondaines.

Venait ensuite l'énumération des devoirs féminins d'épouse, de mère et de maîtresse de maison. Les membres de la confrérie devaient viser à la perfection en imitant les vertus de la Vierge et en incitant leurs enfants à imiter Jésus, leur mari, saint Joseph et leurs domestiques les saints anges.

Tout manquement grave aux règlements de la confrérie pouvait entraîner l'exclusion. Le motif déterminant consistait "dans les péchés scandaleux qui pourraient faire décrier la Sainte-Famille mais non pas pour des vices secrets et cachés».

c) Recrutement des prêtres et des religieuses

Pour accentuer le rôle d'élite morale de la confrérie de la Sainte-Famille, $\mathbf{M}^{\mathrm{gr}}$ de Laval invita les membres des communautés religieuses à s'y inscrire. Ceux-ci répondirent à son appel et la confrérie compta parmi ses membres 209 prêtres et religieuses.

\section{COMPOSTTION DE LA CONFRÉRIE DE LA SAINTE-FAMILLE}

$\begin{array}{llrl}\text { Prêtres } & : & 61 \\ \text { Religieuses } & : & 148 \\ \text { Étrangers } & : & 91(14 \text { h. } 77 \mathrm{f} .) \text { de Bourgogne. } \\ \text { Laïcs canadiens } & : & 985(51 \mathrm{~h} .934 \mathrm{f} .) \\ \text { Indiens } & : & 78(14 \text { h. } 64 \mathrm{f} .) \\ \quad \text { Total } & : & 1,363 \text { personnes. }\end{array}$

\section{B - Élite sociale à la tÊTe de la confrérie}

Une analyse détaillée de l'origine sociale des 934 dames de la Sainte-Famille révèle que toutes les catégories sociales étaient représentées au sein de la confrérie.

Les membres du Conseil de direction appartenaient également à tous les groupes sociaux. Leur âge et leur ancienneté au sein de la confrérie constituaient les facteurs déterminants de leur élection. Pour les années 1744-1763, l'âge moyen des conseillères était de 58.5 ans et leur ancienneté moyenne de 21.7 ans. Cela 
dénote une tradition bien établie d'élire des personnes d'âge mûr ayant donné des preuves de leur vertu et de leur piété.

Les supérieures de la confrérie étaient elles aussi des personnes âgées, anciennes dans la confrérie, ayant occupé un poste de conseillères avant d'être élues supérieures. Mais en plus, la supérieure de la confrérie appartenait toujours à l'élite sociale de la colonie. Sur les 5 supérieures connues de la confrérie de Québec, trois étaient des épouses de gouverneurs (Louis d'Ailleboust, Jean Bourdon, Pierre de Rigaud de Vaudreuil de Cavagnial), une, la femme d'un chirurgien éminent, marguillier de Québec (Timothée Roussel) et la dernière, la femme d'un homme d'affaires très important, Jean-Baptiste Poulin de Courval, également constructeur de bateaux, seigneur et procureur du roi.

On peut donc définir ces supérieures comme des personnes alliant une vertu irréprochable à une haute situation sociale.

C'était probablement l'intention de $\mathrm{M}^{\mathrm{gr}}$ de Laval d'augmenter l'influence de sa confrérie en regroupant une élite sociale en même temps qu'une élite morale. Il s'efforça donc de recruter le plus grand nombre possible de dames de la haute société. De 1664 à 1685 , i.e. durant son vicariat apostolique et son épiscopat, la confrérie accueillit 11 femmes de gouverneurs (sur un total de 12), 18 femmes de conseillers (sur 31), 40 femmes de seigneurs (sur 68) et la seule femme d'intendant, Madeleine de Chaspoux de Champigny, cousine de $\mathrm{M}^{\mathrm{gr}}$ de Laval, en 1688. $\mathrm{M}^{\mathrm{gr}}$ de Laval espérait avec raison que l'exemple donné par ces dames de la haute société aurait un effet d'entraînement sur le reste de la population.

Néanmoins, quels que soient la haute situation sociale ou le poste élevé occupé par une dame au sein de la confrérie, elle pouvait se voir obligée de se retirer si elle se permettait le moindre écart de conduite. C'est ce qui arriva à $\mathbf{M}^{\mathrm{me}}$ De Feltz, supérieure de la confrérie de Montréal, invitée à démissionner parce qu'elle avait assisté à un bal. Madame Elisabeth Bégon, belle-sœur de l'intendant, fut elle aussi expulsée sans ménagement parce qu'elle avait participé à un charivari.

Les directeurs de la confrérie, i.e. l'évêque et les curés, entendaient privilégier l'élite morale plutôt que sociale et lorsque la 
bonne réputation de l'organisme était en jeu, ils ne se permettaient pas la moindre concession à l'esprit du monde.

\section{C-Augmentation des efFectifs}

Jusqu'en 1676, la confrérie de la Sainte-Famille fut réservée aux femmes mariées, mais à partir de cette date, $\mathbf{M}^{\mathrm{gr}}$ de Laval décida d'admettre aussi les jeunes filles. Recrutées en majorité parmi les élèves des ursulines et la progéniture des dames déjà associées, ces nouvelles recrues s'habitueraient très jeunes à respecter les règles sévères de la confrérie et assureraient une relève pour l'avenir.

Cet élargissement du recrutement permit l'admission de quelques jeunes filles qui par la suite ne firent pas tout à fait honneur à la confrérie. Comme Jeanne Picoté de Belestre, qui devint plus tard la maîtresse de Pierre Le Moyne d'Iberville et Louise Guyon, mieux connue sous le nom de $\mathrm{M}^{\text {me }}$ de Freneuse, qui fut pendant plusieurs années la maîtresse de Simon-Pierre Denys de Bonaventure, en Acadie.

Les règlements de la confrérie de la Sainte-Famille, les conditions d'admission restèrent les mêmes tout au long du Régime français. Le recrutement connut cependant des fluctuations considérables, comme pour les deux autres confréries du Rosaire et du Scapulaire.

\section{II - ÉVOLUTION DU RECRUTEMENT \\ DE LA CONFRÉRIE DE LA SAINTE-FAMILLE}

Le type de dévotion austère que la confrérie de la SainteFamille imposait à ses membres reflétait assez bien le climat religieux de la Nouvelle-France au $\mathrm{XVII}^{\mathrm{e}}$ siècle. Mais au siècle suivant, le recrutement de la confrérie baissa considérablement parce que sa spiritualité ne correspondait plus à l'atmosphère religieuse du siècle. Cette diminution du recrutement était particulièrement accentuée chez les dames de la haute société et l'âge des membres au moment de l'admission augmentait constamment.

En plus de ces changements majeurs qui se sont produits au tournant du siècle, on peut distinguer quatre phases princi- 
pales dans le recrutement de la confrérie. La période de fondation (1664-79) s'accompagne d'un recrutement intensif, suivie d'une baisse en 1680-1681. Puis la confrérie prend un nouveau départ et son élan se maintient jusqu'en 1703. Une descente s'amorce alors qui se poursuit jusqu'aux environs de 1720 . Le recrutement recommence ensuite et augmente régulièrement jusqu'en 1760 .

La même courbe de recrutement s'applique aux confréries du Rosaire et du Scapulaire.

\section{A-Évolution du ReCrutement lä̈c Canadien}

\section{Phase 1664-1679}

La période de recrutement intensif de la confrérie se produisit à une époque où la dévotion à la sainte Famille atteignait un sommet. La plupart des miracles attribués à l'intercession de la sainte Famille se sont produits entre 1663 et 1666, juste au bon moment pour appuyer l'action du clergé qui s'efforçait de répandre cette dévotion parmi la population.

Les événements de l'époque expliquent cette croyance facile aux interventions surnaturelles. Les habitants de la colonie avaient été terrorisés par les guerres iroquoises et par le tremblement de terre de 1663, présenté comme une punition divine. En 1665, ils considérèrent l'arrivée du régiment de Carignan comme une bénédiction du Ciel. D'où cette recrudescence de piété.

A ce climat de tension nerveuse et d'exaltation religieuse succéda un impérieux besoin de détente. Durant le carnaval d'hiver 1667 , les bals et les réunions mondaines se multiplièrent et les dames de la Sainte-Famille y prirent part comme les autres, ce qui entraîna une sanction immédiate de la part de $\mathbf{M}^{\mathrm{gr}}$ de Laval : il suspendit les réunions de la confrérie. L'intendant Jean Talon, indigné de cette sévérité, demanda au Conseil souverain d'ouvrir une enquête, mais peu de temps après il retira sa requête. Il se rappelait probablement que Colbert lui avait recommandé d'éviter les conflits ouverts avec le clergé.

A la même époque, $M^{\mathrm{gr}}$ de Laval fit admettre quelques hommes dans la confrérie en dépit des règlements qu'il avait luimême promulgués. 
Les plus célèbres de ces confrères associés sont l'intendant Jacques Duchesneau, les conseillers Jean Bourdon, Mathieu Damours de Chauffours, Mathieu Damours de Freneuse, Denis-Joseph de Ruette d'Auteuil, Denis Peuvret Demesnu, Alexandre Peuvret Demesnu et l'explorateur Louis Jolliet.

Ce n'est sûrement pas par hasard que trois de ces conseillers avaient été appelés au Conseil souverain en 1663 à la suggestion de $\mathbf{M}^{\mathrm{gr}}$ de Laval: soient Jean Bourdon, Mathieu Damours de Chauffours et Denis-Joseph de Ruette d'Auteuil. $\mathbf{M}^{\mathrm{gr}}$ de Laval espérait exercer assez d'influence au Conseil, par l'intermédiaire de ce groupe de dévots, pour faire interdire la traite de l'eau-de-vie. Il avait également fait nommer $\mathbf{M}$. de Mésy gouverneur de la Nouvelle-France dans le même but, croyant que ce dévot, exmembre de l'Ermitage de Cäen, accepterait de suivre les conseils de l'évêque.

Cette façon de procéder aboutit à un échec total. M. de Mésy se brouilla avec $\mathbf{M}^{\text {gr }}$ de Laval. L'intendant Duchesneau et les conseillers n'osèrent pas s'opposer complètement à la traite de l'eau-de-vie.

\section{Phase $1680-1703$}

A part ces incidents, les activités et le recrutement de la confrérie se maintinrent à un niveau très satisfaisant jusqu'en 1703. En 1690, lors de l'invasion de Phips, Mgr de Saint-Vallier recommanda à la population une grande dévotion à la sainte Famille, tandis que $\mathbf{M}^{\mathrm{gr}}$ de Laval faisait accrocher un tableau représentant ces trois personnes au clocher de la cathédrale pour protéger la ville contre les boulets anglais.

En 1694, $\mathrm{M}^{\mathrm{gr}}$ de Saint-Vallier ordonna à tous les curés de fonder au moins une confrérie par paroisse. Dans les années qui suivirent, le recrutement augmenta pour les trois confréries du Rosaire, du Scapulaire et de la Sainte-Famille.

$\mathrm{M}^{\mathrm{gr}}$ de Laval et $\mathrm{M}^{\mathrm{gr}}$ de Saint-Vallier pouvaient avoir des vues différentes sur la façon d'administrer le diocèse, mais ils faisaient preuve tous deux de la même dévotion à la sainte Famille et surtout de la même austérité, du même rigorisme moral. Les règlements sévères de la confrérie ne pouvaient que rencontrer l'appro- 
bation de $\mathrm{M}^{\mathrm{gr}}$ de Saint-Vallier qui trouva en elle un appui solide de son affrontement avec Frontenac au sujet des comédies.

Cette fameuse querelle entre le gouverneur et l'évêque est bien connue. En 1693, Frontenac fit jouer Nicomède de Corneille et Mithridate de Racine. L'année suivante, il prépara Tartuffe, mais devant les protestations de l'évêque et, en échange d'espèces sonnantes et trébuchantes, il renonça à son projet. Cet incident fait ressortir le rôle social et religieux que les dames de la SainteFamille jouaient en Nouvelle-France. Bertrand de Latour nous éclaire sur cet aspect de la question :

Ce fut M. de Frontenac qui [...] introduisit la comédie en ce pays $[. .$.$] On en fut scandalisé, la plupart des femmes et des$ filles, élevées dans des principes de religion, refusèrent des rôles et ne voulurent pas y assister, surtout celles de la Sainte-Famille établie à la paroisse, qui sont en fort grand nombre et les plus distinguées. Les invitations, les promesses, les menaces du gouverneur que la résistance rendait plus vif, ne purent en gagner que trois qui furent aussitôt exclues de la Sainte-Famille. Il en fut offensé et en usa mal avec l'évêque qui avait ordonné l'exclusion ${ }^{4}$.

Frontenac eut la même réaction que l'intendant Talon dans des circonstances analogues et il réclama une enquête.

Lorsque $\mathrm{M}^{\mathrm{gr}}$ de Laval recrutait les dames de la Sainte-Famille parmi les personnes " les plus distinguées » de la colonie, il utilisait une arme à double tranchant. Cette élite sociale et morale exerçait assez d'influence pour dissuader «la plupart des femmes et des filles " d'assister à la comédie, mais inversément, la réaction du gouverneur (ou de l'intendant) était d'autant plus violente en voyant les femmes des notables refuser ses invitations ou être chassées de la confrérie pour avoir accepté. Lors d'une réception, Frontenac invitait certainement en premier lieu les épouses de l'intendant et des conseillers. Or, elles étaient presque toutes de la Sainte-Famille et ne pouvaient à la fois se rendre à ses invitations et rester dans l'association.

Quelques-uns des contemporains de Frontenac partageaient ses griefs contre le clergé en général et la confrérie de la SainteFamille en particulier. Le récollet Sixte Le Tac écrivait :

4 Louis Bertrand DE Latour, Euvres complètes, Paris, Migne, 1855, IV, p. 57. 
Les Jésuites tiennent tout le pays en servitude et en esclavage. Une si grande sujétion fait paraître à l'extérieur beaucoup de dévotion : pourvu qu'un homme soit dans la Congrégation [...] il est dans les bonnes grâces et à couvert de toutes misères; une femme de même si elle veut être estimée, doit être de la Sainte-Famille 5.

Un autre témoignage du même genre est dû à un auteur anonyme qui signe "un ami de l'abbé de Galinée ».

Il y a dans Québec une congrégation de femmes et de filles qu'ils appellent la Sainte-Famille dans laquelle on fait vœu sur les saints évangiles de dire tout ce qu'on sait de bien et de mal des personnes qu'on connaît [...] La Compagnie s'assemble dans la Cathédrale à porte fermée et là elles se disent les unes aux autres ce qu'elles ont appris. C'est une espèce d'inquisition contre toutes les personnes qui ne sont pas unies avec les jésuites. Ces personnes sont accusées de tenir secret ce qu'elles apprennent de mal des personnes de leur party, et de n'avoir pas la même discrétion pour les autres ${ }^{6}$.

Il convient de garder beaucoup de réserve vis-à-vis ces auteurs qui veulent visiblement dénigrer les jésuites. Même si le clergé faisait preuve d'un zèle parfois indiscret, rien ne prouve que la confrérie tyrannisait le reste de la population.

\section{Phase $1703-1720$}

Après 1703, le recrutement de la confrérie amorce une phase descendante qui ne s'arrête qu'en 1720 . Un phénomène identique se produit pour les confréries du Rosaire et du Scapulaire. À quoi faut-il attribuer cette incidence? Peut-être au décès de $\mathbf{M}^{\mathrm{gr}} \mathrm{de}$ Laval en 1708 et au séjour prolongé de $\mathrm{M}^{\mathrm{gr}}$ de Saint-Vallier hors de la colonie de 1704 à 1713.

Les dames de la Sainte-Famille firent très peu parler d'elles au cours de ces deux décennies. Seule l'invasion de Walker en

5 Sixte LE TAC, Histoire chronologique de la Nouvelle-France depuis sa découverte jusques en l'an 1632. Publié d'après le manuscrit original de 1689 par Eugène Réveillaud, Paris, Fischbacher, 1888, pp. 27-28.

6 Pierre Margry, Découvertes et établissements des Français dans l'ouest et dans le sud de l'Amérique septentrionale. Mémoires et documents inédits, Paris, Maisonneuve, 1879; I : p. 370. 
1711 leur fournit l'occasion de déployer leur ferveur religieuse, multipliant les neuvaines, les processions et les vœux.

L'atmosphère religieuse du Canada en ce début du XVIII ${ }^{\mathrm{e}}$ siècle apparaît paisible et d'une ferveur modérée. Les querelles bruyantes entre les puissances temporelles et spirituelles appartiennent au passé aussi bien que les miracles spectaculaires ou les élans mystiques.

\section{Phase 1720-1760}

Après 1720 , le recrutement de la confrérie reprend de la vigueur et continuera d'augmenter doucement jusqu'en 1760. Les confréries du Scapulaire et du Rosaire profitent de la même remontée. L'intendant Gilles Hocquart décrit le climat religieux de cette époque en ces termes : "Tous sont communément attachés à la religion ${ }^{7}$ ".

En 1755, le curé de Québec, Félix Récher, nomma présidente honoraire de la confrérie, l'épouse du gouverneur Vaudreuil. La présence de la première dame de la colonie à la tête de la confrérie produisit l'effet d'entraînement que le curé espérait et le recrutement doubla cette année-là. Madame de Vaudreuil exerça aussi une influence modératrice sur la vie sociale de la colonie. Le témoignage de Montcalm en fait foi. Décrivant les activités mondaines des officiers français, il ajoute qu'il y en aurait eu beaucoup plus «si le goût de $M$. et $\mathbf{M}^{\text {me }}$ de Vaudreuil n'était tourné vers la dévotion ${ }^{8}$ ».

A Montréal, un dernier incident se produisit lorsque plusieurs dames de la confrérie se virent contraintes de se retirer parce qu'elles avaient assisté aux bals donnés en l'honneur de l'intendant Bigot. Madame Bégon rapporte ces faits avec inquiétude. Elle craint de voir des excommunications. Mais le XVII ${ }^{\mathrm{e}}$ siècle religieux est loin derrière et l'intendant n'a aucune envie de chercher noise au clergé pour pareille bagatelle.

7 Gilles HocQuart, Mémoire du 30 octobre 1737, C 11 A, Vol. 67, f. 95 .

8 Louis-Joseph DE MontCalm, Journal du marquis de Montcalm durant ses campagnes en Canada de 1756 à 1759, publié sous la direction de l'abbé Henri Casgrain, Québec, Demers et Frère, 1895, p. 154. 


\section{B-Évolution du RECRUTEMENT dANS LA HAUTE SOCIÉTÉ}

Ces sanctions décrétées en 1749 montrent bien que le clergé demeurait aussi sévère qu'en 1667, mais la population dans son ensemble répondait moins bien à ses exigences.

Sur un total de 985 laïs canadiens inscrits dans les registres de la Sainte-Famille, $62.9 \%$ ont été admis au XVII ${ }^{\mathrm{e}}$ siècle, contre $37.4 \%$ seulement au XVIII ${ }^{\mathrm{e}}$. Cela malgré l'augmentation de la population.

Cette diminution du recrutement est particulièrement accentuée chez les groupes élitistes. (Par groupes élitistes, j'entends les femmes des gouverneurs, intendants, conseillers et seigneurs.) $80.3 \%$ de ces dames de la haute société ont été recrutées au $\mathrm{XVII}^{\mathrm{e}}$ siècle; seulement $19.6 \%$ au XVIII .

RECRUTEMENT DES DAMES DE LA HAUTE SOCIÉTÉ

\begin{tabular}{ccccc}
\hline & $\begin{array}{c}\text { Femmes de } \\
\text { seigneurs }\end{array}$ & $\begin{array}{c}\text { Femmes de } \\
\text { conseillers }\end{array}$ & $\begin{array}{c}\text { Femmes de } \\
\text { gouverneurs, } \\
\text { intendant }\end{array}$ & Total \\
\hline $1664-99$ & 54 & 24 & 12 & 90 \\
\hline $1700-30$ & 9 & 3 & 0 & 12 \\
\hline $1731-60$ & 5 & 4 & 1 & 10 \\
\hline TotaL & 68 & 31 & 13 & 112 \\
\hline
\end{tabular}

La cause de ce double phénomène de diminution du recrutement réside probablement dans la sévérité excessive des règlements de la confrérie. Les dames de toutes les classes sociales, mais plus particulièrement celles de la haute société, trouvaient sans doute trop dur de devoir renoncer à toute vie mondaine.

Le clergé qui dirigeait la confrérie, lui, se refusa à toute concession. Il aimait mieux voir diminuer le recrutement que d'atténuer la rigueur des règlements. Les dames qui continueront de s'inscrire à la confrérie seront moins nombreuses, mais elles constitueront un groupe de dévotes convaincues. Mieux valait la qualité que la quantité. Regrouper une élite morale était plus important que recruter toute l'élite sociale. 
C-Évolution de LA COURBE D'ÂGE

Les dames en nombre plus restreint qui adhérèrent à la confrérie au XVIII ${ }^{e}$ siècle étaient en même temps plus âgées que celles qui s'inscrivaient au XVIIe.

Entre 1676 et 1699, seulement $10.3 \%$ des femmes ont plus de 40 ans au moment de leur inscription. En 1731-1763, 60.2\% sont âgées de plus de 40 ans.

Ce vieillissement de la confrérie s'explique par le fait que les dames plus âgées trouvaient moins difficile que les jeunes de renoncer à tout divertissement.

La baisse du recrutement de la confrérie de la Sainte-Famille au XVIII e siècle et le vieillissement de ses membres ne traduisent pas forcément une diminution de la ferveur religieuse au XVIII ${ }^{\mathrm{e}}$ siècle. Il s'agit plutôt «d'un changement dans le contenu des dévotions », selon l'expression de Michel Vovelle 9.

\section{D - Évolution DU RECRUTEMENT SELON LES SEXES}

Tandis que le recrutement de la Sainte-Famille décroît, celui du Scapulaire et du Rosaire se maintient. Mais une autre évolution se dessine à l'intérieur de ces deux confréries qui concerne l'appartenance sexuelle des personnes inscrites. En effet, au XVII' siècle, la majorité des membres des confréries du Scapulaire et du Rosaire sont des hommes. Au XVIII siècle, par contre, la majorité est féminine.

La prédominance masculine au sein des confréries au XVII siècle peut s'expliquer par le fait que les hommes étaient plus nombreux dans la colonie. L'équilibre des sexes fut atteint vers 1720 , mais après cette date, les femmes deviennent beaucoup plus nombreuses que les hommes dans les confréries. Pour la période 1726-1760, la confrérie du Rosaire reçoit $16.9 \%$ d'hommes contre $82.2 \%$ de femmes (avec $0.9 \%$ d'inconnus). Le Scapulaire accueille $29.8 \%$ d'hommes et $61.9 \%$ de femmes ( $8.3 \%$ d'inconnus). Cela permet de conclure à une véritable féminisation de la dévotion, au sein des confréries du Scapulaire et du Rosaire tout au moins.

9 Michel Vovelle, Piété baroque et déchristianisation en Provence au XVIIIe siècle, Paris, Plon, 1973, p. 497. 
Les spécialistes en sociologie des religions ont fréquemment constaté ce phénomène de "dimorphisme sexuel ${ }^{10}$ " en matière de pratique religieuse. Presque partout, les femmes se montrent plus attachées que les hommes aux signes extérieurs de la religion. L'historien Michel Vovelle exprime l'avis que «la féminisation des dévotions au XVIIIe siècle [...] semble bien être une attitude historiquement élaborée que l'on voit naître alors ${ }^{11}$ ».

La simultanéité de ce phénomène en France et en NouvelleFrance permet de penser que des relations très suivies existaient entre la colonie et la mère-patrie.

\section{CONCLUSION}

Tout au long du Régime français, la confrérie de la SainteFamille a représenté le type de dévotion idéal que le clergé souhaitait voir pratiquer.

L'esprit de la confrérie fut défini par $\mathbf{M}^{\mathrm{gr}}$ de Laval et il évolua fort peu par la suite. Son caractère essentiel resta toujours le même : constituer un corps d'élite moral qui prêcherait par l'exemple face au reste de la population.

Le caractère théocentrique de la dévotion à la sainte Famille, le grand effort d'intériorisation qu'elle demandait, ses exigences morales ne destinaient pas la confrérie à un recrutement de masse. $\mathrm{M}^{\mathrm{gr}}$ de Laval l'avait fort bien compris quand il l'avait organisée sur le modèle de la Congrégation de la Vierge réservée à un nombre restreint de dévots soucieux de sanctification et de perfectionnement personnels.

A côté de la haute spiritualité pratiquée au sein de la confrérie, une forme popularisée de la dévotion à la sainte Famille se répandit parmi la masse populaire. Les soldats du régiment de Carignan qui récitaient le chapelet de la Sainte-Famille, à l'instigation du père Chaumonot, participaient à cette dévotion sans être affiliés à la confrérie. Et quand $\mathbf{M}^{\mathrm{gr}}$ de Saint-Vallier recommande à tous ses diocésains une grande dévotion à la sainte Famille, en 1690 , il ne s'adresse pas aux seuls membres de la confrérie.

Confronté avec le problème de baisse du recrutement de l'association, le clergé refusa absolument de diminuer les exigences $\mathrm{du}$ règlement. Cette attitude intransigeante eut pour effet de 
conserver intacte la spiritualité de la confrérie qui constitua jusqu'à la fin une école de sainteté pour les plus fervents de ses membres. Marguerite d'Youville en est le dernier et le meilleur exemple.

De $M^{g r}$ de Laval à $M^{g r}$ de Pontbriand, la confrérie de la Sainte-Famille réussit à exercer une influence modératrice sur la vie mondaine de la colonie et à donner à une partie de la société canadienne le ton de la dévotion.

\author{
Marie-Aimée Cliche \\ École des gradués (histoire), \\ Université Laval, Québec.
}

10 Gabriel LE Bras, Études de sociologie religieuse, Paris, P.U.F., 1955, pp. 356-357.

11 Michel Vovelle, op. cit., p. 133. 